\title{
Intellicor: Mobile Design for Monitoring Phonographic Signals
}

\author{
Malin WIST JAKOBSEN ${ }^{\mathrm{a}, 1}$ and Ankica BABIC ${ }^{\mathrm{b}}$ \\ ${ }^{a}$ Department of Information Science and Media Studies, University of Bergen, Norway \\ ${ }^{\mathrm{b}}$ Department of Biomedical Engineering, Linköping University, Sweden
}

\begin{abstract}
A mobile and web-based prototype was developed to explore utility of heart sound data in the context of patient self-monitoring. There are not many applications available despite measurement equipment that can be purchased. This research aimed at developing an application that could help patients understand and use phonocardiography. The resulting prototype Intellicor enables easy-to-use web and mobile solutions such as registering heart sound, review of previous heart signal recordings, summaries of terms related to patient condition, and medication taken. These functions can be utilized by both patients and physicians to create understanding of heart signals and build communication as a part of treatment. Three development iterations included several expert evaluators who gave positive feedback on the concept of the application. It was appreciated that patients could monitor heart signals and better understand the results. The medical experts would welcome such a system into their work domain if developed correctly and in accordance with the formal expectations, both legal and clinical. The prototype has shown the advantage of gathering data otherwise impossible to obtain. The Intellicor prototype presents the foundation that ought to be further developed in close cooperation of clinical and biomedical experts. The self-monitoring of this kind could benefit patients and the healthcare sector as demonstrated by the Intellicor prototype.
\end{abstract}

Keywords. Heart signals monitoring, Phonocardiography (PCG), application, eHealth system, expert evaluation

\section{Introduction}

Life changes due to complication of heart signals affect a large part of the Norwegian population, therefore indicating needs for monitoring of heart signals to a greater extend. There are medical indications, such as early symptoms of heart attacks, angina, and heart failure. As many as approximately 40000 Norwegians annually receive specialist healthcare services related to heart attacks or angina, and 16000 for heart failure [1]. People also monitor themselves remotely to track their behavior and workouts to get the best possible health benefits. Medical staff is also interested in patient self-monitoring due to the large number of patients they need to follow up, so they also recognize the advantage of remote self-monitoring without the intermediate help of health personnel.

Mobile and web applications can show signal activity to help users determine how and when to monitor their hearts and receive information about when to contact medical personnel. Many patients struggle with basic instructions and information from their

\footnotetext{
${ }^{1}$ Corresponding Author, Malin Jakobsen; E-mail: malin@j-j.no
} 
health personnel due to limited health literacy and strive to know what to do and how to interpret findings when they self-monitor [2]. Smart phones already offer some applications such as Samsung health that contribute to physical fitness and health. Kardia is a mobile application for monitoring ECG signals [3]. There are not many applications for monitoring heart sound, even though measurement equipment is available such as electronic stethoscopes that can be combined with application to monitor heart signals.

\section{Method}

The design science research [4] paradigm was utilized to create an artifact through three design iterations. The design solutions ranged from low to high fidelity and were tested by relevant stakeholders and user groups. Stakeholders for this artifact are health personnel who could help patients monitor their health remotely and make sense of their measurements using mobile-web solutions. The second stakeholders are the patients who will monitor themselves using mobile web applications. One more potential stakeholder is a patient's family member who could assist the patient to self-monitor when necessary. Additional stakeholders can be found in the form of insurance agencies, health systems and society at large. Due to the corona virus pandemic at the time of the research these groups were not involved in the development but will be considered in the future.

The artifact's goal and context were to show proof of concept by building a prototype and testing it with today's technology which included development and evaluation tools.

The evaluation was conducted in two parts: system requirements for the prototype addressing both patient and physician needs using semi-structured interviews with field experts. Standardized evaluation methods such as Nielsen's heuristics, system usability scale and system usability testing were applied by usability experts.

\section{Results}

\subsection{The Artifact}

The artifact has two views, one enabling patients to remote monitor heart signals, and one for physicians who intend to utilize the data gathered from remote monitoring as a consultation tool (Figure 1, left side). The functionalities available to the patients are measuring heart signals with the use of PCG method, contact information to medical personnel, overview of earlier PCG measurements and medication overview to help patient manage and remember their medication. To share the data with medical personnel a generate QR code functionality was implemented. Similar functions are already seen in existing medical application available on the market, but currently are not used in Norway. As compared to earlier interpretations of PCG measurements, the patient will have a more understandable overview of their own heart signals, which helps elevate the significant problems surrounding health literacy that many adults face [5].

Based on the feedback gathered from the expert interviews new functionalities were added in the second iteration of the prototype. The result was a physician's view (Figure 1, right side) with the following functionalities: Registering QR code for transmitting patient data, overview of patients and their earlier recordings, encyclopedia with terms related to patients, and overall statistics over patients and their health history. 


\subsection{Evaluation of the Intellicor prototype}

First understanding of expert expectations regarding the utility came from conducted interviews with a medical and a biomedical expert. This way, the Intellicor prototype development was informed about how medical personnel would use such a system and what they would expect to have for a consultation.

For PCG to be appropriately done, the article will propose the following key points to be considered when creating such an application for the medial domain:

- Good training for the individuals who are going to use the application,

- Good, but simple results view of heart sounds,

- Good feedback to the user regarding the quality of the signal,

- Statistical feedback regarding measurement. The results also should indicate the probability of having certain symptoms and an explanation about those.

A total of eight participants evaluated the third and final prototype using evaluation tools such as Nielsen's heuristic evaluation and System Usability Scale. In addition, system usability tasks were designed (Figure 2). Six of the participants were usability experts and two were medical experts with little evaluating experience. The average resulting usability score was 90 , which indicates high users' satisfaction, confirmed also through observations and comments.

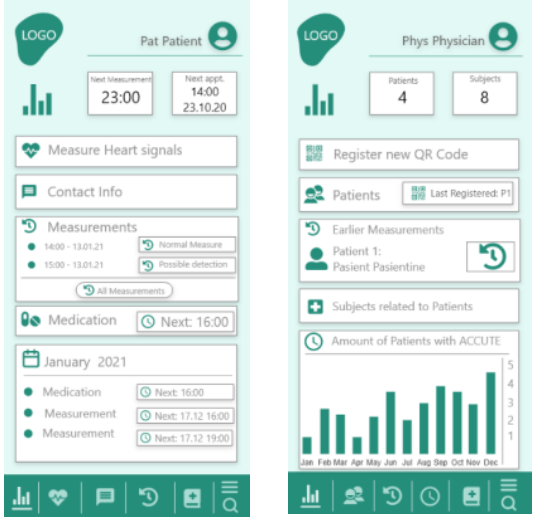

Figure 1. Mobile view of the prototype, patient view (left) and physicians view (right)

Patient
$\begin{aligned} & \text { Start measuring and look at the results } \\ & \text { Navigate to the main screen }\end{aligned}$
$\begin{array}{lll}\text { Find settings and log out } & \text { Register patient results } \\ \text { Navigate to the information screen } & \text { Navigate to the main screen } \\ \square \text { Show earlier measuring results } & \text { Navigate to the information screen } \\ \text { You are wondering about a medical terminology, } & \square & \text { Show earlier measuring results } \\ \text { how would you find more information regarding } \\ \text { that term }\end{array}$

Figure 2. System Usability Tasks 


\section{Discussion}

The main goal of creating the Intellicor prototype was to understand how feasible it would be for patients to monitor and record their heart sounds (PCG). Throughout all three development iterations efforts were made to secure a better understanding of medical content, especially following interaction design principles in the health-care sector. A key focus during evaluations and interviews was to address user needs, how potential users would want to have their health data presented. It is not often discussed during the patient consultation, but it could be assumed that patients are willing to selfmonitor and share the data with healthcare personnel since there were already some commercial systems available for such purposes. However, Norwegian law sets clear and high demands regarding data sharing and privacy which was acknowledged in the development of the Intellicor prototype; no online consultations would be allowed, only collecting data, and transmitting it using QR code.

An assessment was conducted to better understand the patient's health situation and the requirements. The heuristic evaluation was instrumental in shaping different perspectives of two major user groups contributing to the iterative development. The interviews and the evaluation sessions with the participants resulted in positive feedback suggesting that an applied combination of medical informatics and user-centered design could tune into the patient needs. System usability was tested by performing a set of usability tasks returning a score of 90 . Such a high usability score could be considered as a good indication to invest in further development by proper national authorities and other stakeholders.

\section{Conclusion}

Through research, interviews, and prototyping, it was possible to include user experience in designing of Intellicor prototype for patient to self-monitor heart signals. Intellicor prototype prospectively gathers data otherwise impossible to obtain and benefit from. Further development involves patients in their real environment.

\section{Acknowledgements}

The authors are thankful for the field experts; Dr. Henrik Holmstrøm, Dr. Arash Gharehbaghi, Dr Constance Weismann, Tuva Tronbøl for insight and feedback.

\section{References}

[1] Ariansen I, Selmer R, Graff-Iversen S, Egeland GM, Sakshaug S. Cardiovascular disease in Norway [cited 2020 Mar 23]; Available from: https://www.fhi.no/en/op/hin/health-disease/cardiovasculardisease-in-norway-

[2] Graham S, Brookey J. Do Patients Understand? Perm J. 2008;12(3):67.

[3] Hjem - ibeat.no [Internet]. [cited 2020 Apr 1]. Available from: https://ibeat.no/?v=c2f3f489a005

[4] Hevner AR, March ST, Park J, Ram S. Design Science in Information Systems Research. Management Information Systems Quarterly. 28. 75- 2004 [cited 2020 Sep 30].

[5] Wali H, Grindrod K. Don't assume the patient understands: Qualitative analysis of thechallenges low health literate patients face in the pharmacy. Res Soc Adm Pharm. 2016 Nov;12(6):885-92. 